\title{
Financiamiento de las campañas a la cámara de diputados en Chile 2017: estimación de factores explicativos
}

\section{Campaign finance in the 2017 chamber of deputies elections in Chile: estimation of explanatory factors}

\author{
Rafael Hernández Araya ${ }^{1}$ \\ Investigador independiente
}

\begin{abstract}
RESUMEN En el año 2017 debutó el nuevo sistema proporcional para las elecciones parlamentarias en Chile, en conjunto con las nuevas reglas sobre financiamiento de campañas. Se proponen tres modelos de regresión lineal que toman como variable dependiente el gasto de las candidaturas, aportes de los partidos, y aportes propios, estimando el efecto de la incumbencia, densidad poblacional, participación en elección pasada, coalición y sexo. Se halla que los incumbentes gastan más, lo que es transversal a todas las coaliciones, y que la coalición Chile Vamos incurre en gasto sustantivamente mayor que sus competidores. Además, los candidatos de la Nueva Mayoría recurrieron más al financiamiento desde sus partidos, en comparación al resto de las coaliciones. La densidad poblacional no es significativa, y la participación en 2016 incide en los aportes propios. Las mujeres gastaron levemente menos que los hombres y realizaron menos aportes propios, pero los partidos donan indistintamente del sexo.
\end{abstract}

PALABRAS CLAVE Gasto electoral; aportes a campañas; elecciones parlamentarias; incumbentes; financiamiento de la política.

ABSTRACT In 2017 the new proportional system made its first appearance for the legislative elections in Chile, along with the new rules for campaign financing. Three linear regression models are proposed taking campaign expendi-

1. Rafael Hernández Araya. MSc Public Policy and Administration (London School of Economics, 2018). Investigador independiente. E-mail: rafael.hernandez.a@alumni.lse.ac.uk. 
ture, contributions from parties, and self-funding as dependent variables, and estimating the effects of incumbency, population density, turn-out in the last election, coalition and sex. Incumbents are more prone to expenditure across each coalition, and Chile Vamos spends markedly more than its competitors. Additionally, candidates from Nueva Mayoría depended more on contributions from their parties in comparison to other coalitions. Population density has no effect, although turn-out in the last election has a positive effect on selffunding; women spent slightly less than men, and less from their own income, although gender is not significant for party financing.

KEYWORDS Electoral spending; campaign funding; congressional elections; incumbents; political financing.

\section{Introducción}

En noviembre de 2017 debutaron las nuevas reglas para las elecciones parlamentarias en Chile. La novedad radica en la aplicación de un nuevo sistema electoral que viene a reemplazar al antiguo binominal, y en la reforma al esquema de financiamiento del gasto electoral. El proceso se caracterizó por la apertura del sistema político a nuevos partidos o fuerzas políticas ${ }^{2}$, una participación electoral menor a la mitad del padrón electoral, la disminución de la propaganda electoral en las calles y la supresión de los aportes de empresas a las candidaturas.

En América Latina, la literatura que analiza el financiamiento de la política y sus resultados electorales es más bien escasa ${ }^{3}$. Los estudios de financiamiento electoral se concentran mayormente en Estados Unidos; además, el canon ha apuntado principalmente a estudiar los efectos del gasto sobre los votos obtenidos, y no tanto en los factores que explican tal o cual magnitud en el gasto.

Ya se han publicado algunos trabajos que analizan la elección de $2017^{4}$. Sin embargo, hacen referencia a variables contextuales y a cómo los resultados de los comicios impactan en el sistema político, en un sentido amplio. Sin embargo, aún no hay análisis académicos sobre el financiamiento electoral. El presente trabajo busca contribuir al entendimiento de por qué las candidaturas gastan lo que gastan, y cómo se financian, tomando como estudio de caso las elecciones de 2017 para la Cámara de Diputados de Chile. Para esto, se proponen tres modelos de regresión lineal multivariada tomando como variable de salida el gasto electoral, aportes de partido y aportes propios. Los datos se obtienen de fuentes oficiales y públicas, y se procesan a través de software de análisis estadístico.

2. BUNKER (2018).

3. MORALES Y PIÑEIRO (2010).

4. TORO y VALENZUELA (2018); BUNKER (2018); FUENTES (2018). 
En la primera sección se repasa la literatura relevante, abordando los aportes más significativos, una revisión de experiencias extranjeras, y se aborda qué se ha escrito al respecto para el caso de Chile. Seguidamente, se caracteriza la nueva institucionalidad que rige las elecciones en Chile y sus diferencias con el modelo anterior; posteriormente, se exponen los resultados de la elección. Luego, son presentados el diseño de la investigación, el tratamiento de los datos, las hipótesis propuestas, y se discuten los resultados obtenidos. Finalmente, se comentan las principales conclusiones.

\section{Breve estado del arte sobre financiamiento electoral}

A nivel comparado, existe profusa literatura sobre gasto electoral, especialmente en aplicaciones al caso de Estados Unidos. Tradicionalmente, el diseño de investigación busca evaluar posibles vínculos entre el gasto de campaña y los outcomes electorales; en este tipo de trabajos la hipótesis a evaluar es la potencial existencia de una relación directa entre mayor gasto electoral y mayor votación. Por otra parte, existe también una literatura mucho menos vasta, en la que el diseño de la investigación considera al gasto electoral como variable dependiente en función de variables explicativas como incumbencia, género, obligatoriedad del voto, entre otras.

Respecto al primer tipo de estudios, valiéndose del uso de estimaciones de mínimos cuadrados ordinarios (OLS, por sus siglas en inglés), las investigaciones empíricas inicialmente indicaban que el efecto del gasto electoral era marcadamente diferenciado para incumbentes y desafiantes. Esta premisa derivaba del trabajo de Gary C. Jacobson ${ }^{5}$, quien usando modelos de regresión realizó un seguimiento de cuatro elecciones consecutivas a la Cámara de Representantes de Estados Unidos, arribando a la conclusión de que mayor gasto electoral por parte de los incumbentes virtualmente no tiene ningún efecto en la votación obtenida. Una posible explicación sugiere que los incumbentes explotan los extensivos recursos de información disponibles para los miembros del Congreso, saturando a los votantes con información sobre sí mismos, antes de que la campaña comience formalmente. Por tanto, al iniciarse la campaña y, por ende, al empezar a computarse el gasto electoral, el efecto marginal de un peso gastado produce retornos en las preferencias del votante muy modestos; por el contrario, los desafiantes comienzan la campaña sin tener mayor presencia, por tanto, cuentan con mayor espacio para darse a conocer ante el electorado, y como consecuencia de ello el rendimiento del gasto electoral es mayor. Es decir, por cada peso gastado, el retorno en términos de conocimiento por parte del electorado es mayor, y su efecto potencial sobre la votación obtenida también es mayor ${ }^{6}$.

5. JACOBSON (1974; 1980; 1984 y 1985).

6. JACOBSON (1990). 
Sin embargo, aquella aproximación no ha estado exenta de críticas. Green y $\mathrm{Krasno}^{7}$ exponen una falencia elemental que haría subestimar el efecto del gasto de los incumbentes: la calidad del candidato. Cuando se controla por la calidad del candidato -atractivo electoral y habilidades en campaña-, la diferencia en el rendimiento del gasto electoral entre incumbentes y desafiantes es menor a lo propuesto inicialmente por Jacobson. Además, la inclusión de esta variable permitiría identificar interacciones entre variables explicativas; esto es, el resultado electoral podría estar mediado por el acople entre el gasto y la calidad del candidato. Para incumbentes fuertes el retorno del gasto efectivamente es marginal, pero para aquellos que enfrentan desafiantes competitivos la relevancia del gasto en su performance electoral aumenta ${ }^{8}$.

Así, para el caso estadounidense hay evidencia en favor de la tesis de que el gasto es significativo solo para desafiantes ${ }^{9}$, aunque por otro lado la importancia explicativa de aquel factor en las votaciones habría disminuido, al menos durante las elecciones de los setenta y ochenta en la Cámara de Representantes ${ }^{10}$. Matizando estas conclusiones, Gerber ${ }^{11}$ encuentra que en las elecciones a la Cámara de Representantes efectivamente el gasto de los incumbentes tiene un efecto no significativo, pero en el Senado se vuelve relevante y además se iguala entre incumbentes y desafiantes. Esto tendría lugar puesto que la información que el votante tiene acerca del incumbente y desafiante en las postulaciones al Senado son muy similares, a diferencia de las elecciones a la cámara baja en donde los desafiantes son menos conocidos y por tanto su gasto les reditúa mayor posicionamiento. $\mathrm{Al}$ contrario, Erikson y Palfrey ${ }^{12}$ muestran que el gasto de los incumbentes tiene efectos significativos en la performance electoral, a todo evento. La novedad de este trabajo radica en que, en vez de usar Mínimos Cuadrados Ordinarios, los autores optan por un modelo de ecuaciones simultáneas para estimar parámetros de máxima verosimilitud con información completa; la conclusión a la que arriban es que una menor efectividad del gasto en incumbentes es un proceso acumulativo. Esto quiere decir, que una vez que son elegidos, en las siguientes elecciones en que busquen su reelección los retornos del gasto se vuelven decrecientes.

$\overline{\text { 7. GREEN Y KRASNO (1998). }}$

8. MOON (2006).

9. ABRAMOWITZ (1988); JACOBSON (1985).

10. ABRAMOWITZ (1991).

11. GERBER (1998).

12. ERIKSON y PALFREY (1998). 
Siguiendo con el caso estadounidense, trabajos más recientes han aportado nuevas variables y aproximaciones metodológicas para estimar los efectos del gasto. Por ejemplo, Abbe y Hernsonn ${ }^{13}$ estudian la "profesionalización" de las campañas -la contratación de consultores y asistentes en la campaña para distribuir propaganda correo por correo, por los medios, realizar encuestas, etcétera- a nivel de legislaturas de los estados. Sus resultados apuntan a que la "profesionalización" de las campañas es función del gasto electoral, que en las elecciones sin incumbente (open seat) los candidatos suelen gastar más en este ítem, y que el recurrir a profesionales tiene un efecto positivo en la performance de los desafiantes.

En lo referente a elecciones de jueces, Hall y Bonneau ${ }^{14}$ hallan que el mayor gasto en campaña aumenta la participación electoral. Por su parte, Sparks ${ }^{15}$ investiga, en el contexto de primarias abiertas en California y Washington para definir candidatos a las elecciones parlamentarias generales; los hallazgos indican que el gasto varía dependiendo de si en la primaria compiten candidatos de un mismo partido, o de varios. Cuando se trata de competencia dentro del partido, los desafiantes gastan más y su gasto rinde más del doble en los votos obtenidos, respecto a los incumbentes.

Asimismo, Butcher y Milyo ${ }^{16}$ analizan a lo largo de sucesivas elecciones, y de diferentes estados, límites a cuánto puede donarse a las campañas, y financiamiento público parcial, tienen un efecto marginal en las posibilidades de los incumbentes. Sin embargo, financiamiento público completo y prohibición de donaciones corporativas aumenta significativamente las probabilidades de reelección. Sprick ${ }^{17}$, usando datos sobre las transacciones realizadas con los ingresos electorales y encuestas electorales, constata que el gasto en propaganda y eventos aumenta el apoyo electoral, cambia la composición de aquellos que participan de la elección -aunque no modifica la intención de voto de un candidato a otro-, y que el gasto en propaganda tiene un efecto mayor sobre los votantes poco informados, que se identifican con un partido, y que declaran estar descontentos con el estado de la economía.

\footnotetext{
13. ABBE Y HERNSONN (2003).

14. HALL Y BONNEAU (2008).

15. SPARKS (2018).

16. BUTCHER Y MILYO (2020).

17. SPRICK (2020).
} 
Adicionalmente, existen aplicaciones de este mismo marco de análisis, a otras latitudes. Tal como en la literatura tradicional, en Corea el gasto es más efectivo en las campañas de desafiantes que de incumbentes ${ }^{18}$. Similar es lo acaecido para Irlanda; Benoit y Marsh $^{19}$ hallan evidencia del efecto positivo del gasto electoral en los votos obtenidos, siendo también significativo únicamente para desafiantes. Lo relevante de su trabajo es que explican que en la práctica no es que el gasto de los incumbentes sea menos efectivo, sino que estos cuentan con gasto "escondido" que no es reportado como gasto propiamente tal, en las cuentas oficiales. Con esto, se refieren al tiempo de trabajo del staff a su disposición, reembolsos por viajes, apariciones públicas relacionadas con actividades oficiales, y envío de correo frecuente. Una vez consideradas estas prerrogativas como gasto electoral y calculadas como tal, la efectividad del gasto de los incumbentes se vuelve sustancialmente mayor, logrando equipararse con la de los desafiantes. Por otra parte, Johnson ${ }^{20}$ encuentra que no hay diferencias en los efectos del gasto sobre la performance electoral, para los casos de Brasil, Finlandia e Irlanda; su explicación radica en que la permisividad del sistema de representación proporcional alienta a los desafiantes que tienen capacidades limitadas para desarrollar apoyo electoral, incluso cuando gastan mucho. En las elecciones locales de Israel, BenBassat $e t a l^{21}$ encuentran que no hay relación entre el gasto electoral y la votación obtenida. Para Alemania Fink ${ }^{22}$ halla algo de efecto para las elecciones federales, pero no para las locales; y en Bélgica ${ }^{23}$ el efecto es débil.

Si bien, como se ha mostrado, se han aportado numerosas contribuciones respecto a los efectos del gasto electoral en la votación obtenida, estudios que tomen el gasto como variable dependiente parecen ser menos recurrentes. Para Estados Unidos, Hogan ${ }^{24}$ toma las elecciones en las legislaturas estatales, y encuentra que las leyes sobre financiamiento electoral tienen un efecto significativo en el gasto por candidato: límites a los aportes y financiamiento público disminuyen el gasto de los incumbentes, mientras que el financiamiento público incrementa el gasto de los desafiantes. Además, rasgos de los candidatos y de sus distritos también tie-

18. SHIN et al (2005).

19. BENOIT y MARSH $(2008 ; 2010)$.

20. JOHNSON (2012).

21. BEN-BASSAT et al (2015).

22. FINK (2012).

23. MADDENS y PUT (2013).

24. HOGAN (2000). 
nen impacto. Adicionalmente, examina si es que existen diferencias por género ${ }^{25}$, sin encontrar diferencias significativas entre hombres y mujeres en la cantidad disponible para gastar. Por su parte, para las elecciones locales en Estados Unidos, Weinscheck y Holbrook ${ }^{26}$ hallan que en aquellos gobiernos locales donde el salario del alcalde es superior, los candidatos tienden a gastar más.

En lo referente a Chile, la literatura académica empírica ha abordado el financiamiento electoral para las campañas parlamentarias en variadas ocasiones. Díaz Rioseco et $a l^{27}$ realizan un análisis descriptivo para 24 campañas electorales desplegadas en seis distritos en la Región Metropolitana durante las elecciones parlamentarias de 2005. Valiéndose de la observación participante, realización de entrevistas semiestructuradas con activistas políticos e informantes clave, además de la información pública, se identifica una presencia de mayor gasto electoral en el caso de los incumbentes que de los desafiantes; a pesar de que esta tendencia es transversal a los distintos tipos de distrito, tomando solamente a los incumbentes, aquellos de la coalición derechista Alianza por Chile elegidos en distritos populares tienden a reportar niveles de gasto significativamente mayores que sus pares de la coalición de centro-izquierda "Concertación".

Otro trabajo descriptivo a este respecto corresponde a Agostini ${ }^{28}$. El autor pone de relieve la importancia de la reforma al financiamiento de la política del año 2003, que buscó regular el dinero en las campañas respecto al estado de cosas anterior, en que no había regulación en absoluto; se constata que en el período 2009-2010 hubo un aumento importante en el número de candidatos que recibieron donaciones y levemente en los montos donados promedio, respecto al período 2005-2006. Esto podría ser expresión de un mayor grado de formalización de los aportes, aunque existe una duda razonable sobre la magnitud de dichas donaciones y del gasto que no es declarado formalmente. Además, se halla que para los partidos pequeños el financiamiento fiscal es más sustantivo -como parte de sus ingresos- que para los partidos grandes; adicionalmente, la evidencia presentada muestra que el gasto sí tiene un efecto en la fracción de votos que obtiene un candidato.

25. HOGAN (2007).

26. WEINSCHECK y HOLBROOK (2014).

27. DÍAZ RIOSECO et al (2006).

28. AGOSTINI (2012). 
Los estudios explicativos han abordado el tema tomando el gasto como variable independiente sobre el resultado electoral ${ }^{29}$, o bien considerando el gasto como variable de salida respecto a otros factores ${ }^{30}$. En el primer caso, para las elecciones de 2005 Morales y Piñeiro encuentran un efecto significativo del gasto en el resultado electoral de los incumbentes, a diferencia de la literatura tradicional sobre la materia. Adicionalmente, para aquellos incumbentes con más de un período en el cargo el retorno del gasto resulta aún mayor. Por otra parte, los incumbentes que en la elección anterior ganaron con más comodidad, contrario a lo esperado, reportan mayores niveles de gasto. Para evitar la endogeneidad entre ambas variables, los autores despliegan un modelo de regresión en dos etapas (two stage least square). Por su parte, Navia y Acevedo proponen un método no endógeno para medir el gasto electoral; se trata de considerar el gasto efectivo de cada candidato, como porcentaje del máximo de gasto permitido en su distrito. Tomando las elecciones tanto de diputados y senadores en 2005 y 2009, se observa que el gasto efectivamente tiene un efecto significativo en el resultado electoral, pero menor a lo reportado cuando se usa el gasto del candidato respecto al gasto total, o el gasto por voto, como variable dependiente.

Respecto al segundo tipo de estudios, Johnson se propone analizar el efecto de la incumbencia y de los distintos tipos de contribuciones a las campañas que permite la legislación, sobre el gasto. De sus resultados se infiere que dado el sistema electoral binominal -vigente para ese entonces-, contrario a lo esperado habría una relación inversa entre el gasto del incumbente, y qué tan competitiva es la respectiva elección. Por las mecánicas propias del sistema, la competencia se da principalmente dentro de la coalición, más que respecto a la coalición contraria. Ya que cada una de las listas de las dos principales coaliciones, tiene prácticamente asegurado uno de los dos cupos en el distrito, el competidor es más el compañero de lista que el candidato de la coalición opositora; por tanto, mayor gasto electoral por parte del incumbente es más bien una protección contra la competitividad su propio compañero de lista -aunque se corre el riesgo de endogeneidad entre competitividad y gasto electoral, puesto que la primera puede ser resultado de la segunda, según señalan Navia y Acevedo ${ }^{31}$. Finalmente, Navia e Iturra analizan los componentes del gasto para las elecciones municipales de 2008 y 2012;

29. MORALES y PIÑEIRO (2006); NAVIA y ACEVEDO (2015).

30. JOHNSON (2011); NAVIA e ITURRA (2016).

31. NAVIA y ACEVEDO (2015). 
en este caso, similar a lo hallado por Morales y Piñeiro, los incumbentes gastan más en propaganda que los desafiantes. Por lo demás, en comunas con niveles más altos de educación los candidatos gastan menos en desplazamientos y más en servicios, mientras que en zonas rurales y en comunas con más pobreza se gasta más en desplazamiento y menos en propaganda.

\section{El financiamiento electoral en Chile: nueva institucionalidad}

En el año 2016, con la promulgación de la Ley $\mathrm{N}^{\circ}$ 20.900, tuvo lugar una reforma que modifica sustancialmente el esquema de financiamiento electoral en Chile "al pasar de un modelo de financiamiento principalmente privado y sin control a un modelo principalmente público y con fuertes controles" ${ }^{32}$.

La literatura se ha abocado a investigar las reformas al financiamiento de la política desde una perspectiva public choice; es decir, se busca responder a la pregunta del porqué los incumbentes buscarían restringir para sí mismos los límites y esquemas de financiamiento permitidos ${ }^{33}$. Para el caso de Chile, Fuentes ${ }^{34}$ hace hincapié en los escándalos de corrupción como un factor importante para entender el timing de las reformas. En efecto, hasta antes de 2003 no existía en Chile regulación del financiamiento de la política; solo someramente la legislación prohibía aportes a campañas electorales desde el extranjero, y establecía una franja electoral gratuita en televisión durante el período de candidaturas. Sin embargo, durante aquel año se dieron a conocer casos de corrupción que involucraban a funcionarios de gobierno en pago de sobresueldos y desvío de dineros públicos para financiar campañas electorales. El proceso de formulación de la política de modernización del Estado en Chile durante el año 2003 ha sido profusamente estudiado ${ }^{35}$. En lo medular, tras un acuerdo político-legislativo entre el Gobierno y la oposición, en que se tomó como referencia las propuestas de un influyente centro de estudios -el Centro de Estudios Públicos, CEP- se discutió y aprobó un paquete de medidas legislativas de amplio alcance, que establecía nuevas regulaciones para los procedimientos administrativos (Ley $\mathrm{N}^{\circ}$ 19.880), selección de altos cargos en la administración del Estado (Ley $\mathrm{N}^{\circ} 19.882$ ), financiamiento de la política (Ley $\mathrm{N}^{\circ} 19.884$ ), beneficios tributarios a las donaciones (Ley $\mathrm{N}^{\circ} 19.885$ ), y compras públicas (Ley $\mathrm{N}^{\circ} 19.886$ ), entre otros.

32. FUENTES (2018) p. 111.

33. SCARROW (2004); ABRAMS y SETTLE (2004); EVANS (2007); ANDERSON y THAM (2014); CASTAÑEDA (2018).

34. FUENTES (2018).

35. OLAVARRÍA GAMBI et al (2011); FIGUEROA-HUENCHO et al (2011); TELLO (2011); OLAVARRÍA GAMBI (2012); RAMOS y SCROLLINI (2013). 
Desde el 2003 al 2016 todas las campañas funcionaron bajo aquella regulación. Sin embargo, el sistema adolecía de diferentes problemas: por ejemplo, si bien se establecía un aporte estatal basal pagado como adelanto o bien como reembolso, la mayor parte de los montos incurridos provenían de donaciones privadas. Una parte muy sustantiva de aquellos aportes se otorgaba bajo el carácter de "reservados", con lo que al menos, formalmente, ni el candidato ni el público sabría la identidad del aportante:

Al permitirse incentivos tributarios a las empresas para hacer donaciones, la mayoría de las contribuciones se canalizaron a través de empresas que realizaban donaciones en montos significativos. Como los partidos de derecha tenían mayores vínculos con el sector privado, recibian un mayor número de aportes, en una relación 3:1 respecto de los partidos de centro-izquierda ${ }^{36}$.

Sin embargo, como se ha mencionado, en buena parte de los casos la identidad del aportante sí era conocida por el candidato, por lo que el sistema operaba perpetuando un financiamiento inaccesible para la ciudadanía, pero de fluida comunicación entre empresarios aportantes y políticos financiados.

Durante el año 2014 estalló un nuevo episodio de corrupción, que involucraba al holding empresarial Penta; uno de sus ejecutivos declaró la existencia de un sistema de aportes para campañas del partido de derecha Unión Demócrata Independiente (UDI), en el que se pagaban boletas de honorarios a operadores del partido, por servicios que nunca fueron prestados. Con esto, se disminuía la base imponible y por tanto permitía evadir impuestos, y además los dineros se triangulaban para cubrir los gastos de campaña.

$\mathrm{Al}$ año siguiente, la investigación detectó un sistema de aportes paralelo, a través de la empresa Sociedad Química y Minera de Chile (SOQUIMICH) en el que también a través de la emisión de boletas fraudulentas se derivaban fondos a militantes de partidos de la coalición de centroizquierda: "hacia junio de 2015, poco más del $10 \%$ de los congresistas enfrentaba investigaciones judiciales. De las quince investigaciones, ocho se referían a financiamiento de campañas, cinco se asociaban con casos de corrupción" ${ }^{37}$. Sumado a esto, se descubrió un episodio de negociaciones por compra de terrenos con fines especulativos, que involucraba al hijo de la Presidenta y una posible operación de tráfico de influencias. Con esto, la popularidad de la Presidenta Bachelet comenzó a deteriorarse sistemáticamente, bajando de $54 \%$ al inicio de su gobierno al $24 \%$ a diciembre de $2015^{38}$. Como reacción, el Gobierno convocó a una comisión con el objeto de generar propuestas para combatir la corrupción, cuyo

36. FUENTES (2018) p. 116.

37. FUENTES (2018) p. 118.

38. GAMBOA y SEGOVIA (2016). 
informe final contenía numerosas medidas de reforma a la institucionalidad de los partidos políticos y del financiamiento electoral.

En síntesis, hasta antes de 2016 existía formalmente una regulación para el financiamiento de la política. Sin embargo, lo que en un inicio fue una investigación tributaria, terminó derivando en que los persecutores judiciales durante el año 2014 descubrieran una extensa red de financiamiento privado, secreto e ilegal que operaba al menos desde $2010^{39}$, aunque probablemente incluso con anterioridad, y transversal a la mayor parte de los partidos. Los aportes provenían de numerosas grandes empresas (pesqueras, mineras, entre otras) e importantes grupos económicos. Con estos dineros se financiaban campañas electorales a expensas de la legislación establecida, y por tanto los montos que se declaraban como gasto por parte de las candidaturas eran sustancialmente menores a los que en la práctica efectuaban, por la vía del financiamiento paralelo.

La investigación supuso el procesamiento judicial de un senador y la presentación de antecedentes contra otros varios parlamentarios.

A esto se suma el hecho de que la legislación tampoco disponía de financiamiento para que los partidos cubrieran sus gastos de funcionamiento regular, más allá de las campañas electorales. Es decir, desde el retorno a la democracia en 1990 hasta la legislación de 2016 no se dispuso, durante un cuarto de siglo, de financiamiento fiscal para la actividad de los partidos políticos.

En efecto, la nueva legislación de 2016 suprimió el financiamiento de empresas tanto a candidaturas como a partidos, rebajó ostensiblemente el monto máximo de los aportes de personas naturales a las campañas, y disminuyó a la mitad el límite máximo de gasto electoral permitido. Además, los topes a los aportes sin publicidad de la identidad del donante también se bajaron para evitar riesgo de captura, puesto que con el anterior esquema muchos de los candidatos que más gastaban eran financiados con aportes reservados, pero en la práctica la identidad del donante sí les era conocida. Adicionalmente, se aumenta el aporte fiscal a las campañas, se limita el espacio físico para la instalación de propaganda electoral, y aumenta las atribuciones fiscalizadoras del organismo encargado de las elecciones. Finalmente, se promulgó también una modificación constitucional (Ley $\mathrm{N}^{\circ}$ 20.870) que establece la cesación en los cargos de parlamentario, alcalde, consejero regional y concejal, por infracción grave a las normas sobre gasto electoral.

39. EQUIPO CIPER, "La caja negra de las platas políticas que sacude a la UDI". Disponible en: < https://www.ciperchile.cl/2014/09/30/la-caja-negra-de-las-platas-politicas-que-sacude-a-la-udi/ $\neg$. [Fecha de consulta: 14 de octubre de 2020]. 


\section{Elecciones con las nuevas reglas}

En el año 2014 se reformó el sistema electoral para la elección de diputados y senadores. Adicionalmente, como se ha explicado, el 2015 hubo un cambio al esquema de financiamiento. Las elecciones de consejeros regionales, parlamentarias y presidenciales tuvieron lugar en noviembre de 2017; sin embargo, las nuevas reglas de financiamiento debutaron el año 2016 en las elecciones municipales.

Para las elecciones de alcaldes y concejales de 2016 la mayor novedad fue la participación particularmente baja, de $35 \%$. Si bien, la participación ya venía a la baja producto de la derogación del voto obligatorio, en el año 2012 la asistencia a las urnas fue del $42 \%$ de la población habilitada para sufragar. Cambió también, producto de las nuevas reglas electorales, la composición del financiamiento de las campañas:

Tabla 1. Financiamiento de campañas de alcaldes, 2012 y 2016.

\begin{tabular}{|l|c|c|}
\hline & $\mathbf{2 0 1 2}$ & $\mathbf{2 0 1 6}$ \\
\hline Aportes de los partidos & $9 \%$ & $28 \%$ \\
\hline Aportes de personas & $47 \%$ & $15 \%$ \\
\hline Aporte propio & $10 \%$ & $24 \%$ \\
\hline Aporte fiscal & $34 \%$ & $33 \%$ \\
\hline
\end{tabular}

Fuente: Elaboración propia con datos del Servel.

Se observa una mayor preminencia del aporte de los partidos. Esto, puesto que la reforma dispuso un aumento en la cuantía del anticipo que pueden solicitar los partidos antes de la campaña; de esta forma, los partidos perciben un ingreso inicial (en función de su votación en la elección anterior), y al comienzo del período de campaña lo reparten entre sus candidatos -e independientes que van en su lista en la papeleta-. Del mismo modo, una vez concluido el período eleccionario, los candidatos reciben un reembolso calculado en función de la cantidad de votos obtenidos, que suple aquella parte del gasto no cubierta por el anticipo fiscal. El monto del reembolso también fue aumentado con la reforma, lo que explica el aumento de los aportes propios; así, los candidatos incurren en mayores gastos a cuenta propia -principalmente, a través de créditos con instituciones financieras- pues tienen la certidumbre de que buena parte de aquellos montos, o la totalidad, les será finalmente reintegrado.

Asimismo, aumentó la tasa de reelección. En las elecciones de 2004, 67\% de los alcaldes que compitieron como incumbentes ganaron las elecciones. Para los comicios de 2008 , la tasa fue de $63 \%$, y en el año 2012 el 60\%. Es decir, en tres elecciones la tasa de éxito de los incumbentes bajó 7\%. Sin embargo, en las elecciones de octubre de 2016 de los 289 alcaldes en ejercicio que buscaban su reelección, 210 lo lograron. Esto 
se traduce en una tasa de éxito del $73 \%$ para los incumbentes, revirtiendo la tendencia y volviendo más difícil para los desafiantes competir por un cupo.

Ahora bien, respecto a las elecciones parlamentarias de 2017, los cambios en la institucionalidad resultaron más significativos. En primer lugar, se cambió la fórmula electoral, pasando del antiguo sistema electoral binominal vigente desde 1989 a uno de representación proporcional moderada de tipo D'Hondt (Ley $\mathrm{N}^{\circ}$ 20.840). Las razones que explican la reforma tienen que ver principalmente con que, bajo el antiguo sistema, los partidos o pactos podrían presentar un máximo de 120 candidaturas para la Cámara de Diputados, distribuidas en 60 distritos a lo largo del país con dos candidatos en la lista por cada uno. Esta configuración resultaba particularmente problemática para los grandes partidos de la coalición Nueva Mayoría (Partido Demócrata Cristiano, Partido Socialista y Partido Por la Democracia) ya que no podían competir en todos los distritos y la negociación para acoplar los candidatos de los distintos partidos a lo largo de los distritos era marcadamente difícil ${ }^{40}$. Ahora con un promedio de candidatos por distrito $(M)$ de 5,5, y pudiendo además presentar un máximo de $M+1$ candidatos, la negociación se descomprime y no hay necesidad de omitir candidatos porque la configuración de la lista ya no resulta incompatible.

Los nuevos distritos para la Cámara de Diputados no son exactamente nuevos, en un sentido literal, sino que "fueron producto o de la fusión de dos o más distritos antiguos o de un nuevo dibujo atendiendo la densidad poblacional y los intereses políticos subyacentes" ${ }^{41}$. Consecuentemente, el mapa distrital resultante beneficia más al centro metropolitano y a las zonas extremas, mientras que la pérdida real es de aquellas zonas más pobres ${ }^{42}$. Todos estos factores cambiaron las expectativas de los actores:

el cambio del sistema electoral produjo que al menos 17 diputados decidieran competir al Senado y que otros 19 no volvieran a postular. Es más, para el año 2017 solo 84 diputados (79\%) buscaron la reelección, cifra más baja que los anteriores periodos en que aproximadamente el 9o\% volvía a postular al cargo. Además, de los que repostularon, el 76,2\% fue reelecto, solo cinco puntos menos que en 2001, año más bajo de reelección de incumbentes con el anterior sistema ${ }^{43}$.

La coalición de centroizquierda Nueva Mayoría fue la que cosechó peores resultados, puesto que de los 20 incumbentes que perdieron su reelección, 16 pertenecían a ese pacto. Por su parte, la coalición de derecha Chile Vamos obtuvo los mejores

40. GAMBOA y MORALES (2016).

41. TORO Y VALENZUELA (2018).

42. ALTMAN (2016).

43. TORO y VALENZUELA (2018) p. 224. 
resultados, ya que sus dos principales partidos, Renovación Nacional (RN) y la Unión Demócrata Independiente (UDI) obtuvieron 36 y 31 escaños respectivamente, convirtiéndose en los partidos más exitosos.

Además, con el nuevo sistema se rebaja el umbral para acceder a un escaño, lo que motiva a nuevas fuerzas a entrar en competencia. Es el caso del nuevo partido Evolución Política, que compitió en la lista de Chile Vamos, granjeándose seis diputaciones. Finalmente, la nueva coalición de izquierda llamada Frente Amplio resultó particularmente exitosa; dado que se componía de partidos nuevos, tenían poco acceso a recursos, lo que los obligó a una estrategia "más selectiva y eficiente en la elección de los territorios a competir" 44 , con lo que pudieron acceder a 20 escaños. Finalmente, el Partido Demócrata Cristiano (DC) se escindió de la Nueva Mayoría. Con esto, se formó una coalición electoral llamada Convergencia Democrática con otros partidos menores de izquierda; esto perjudicó marcadamente a la DC, puesto que pasaron de tener 22 diputados y ser la fuerza electoral más importante de la ex-Nueva Mayoría, a tener 14 diputados siendo superados por las otras tres coaliciones. Respecto a las nuevas coaliciones, Frente Amplio y Convergencia Democrática, la obtención de más de $10 \%$ de los votos para cada una de estas dos terceras fuerzas es un hecho histórico inédito. En las elecciones anteriores, cuando el mapa electoral giraba en torno a la Nueva Mayoría y Chile Vamos -Concertación y Alianza, para ese entonces-, ambos pactos se llevaban más del 90\% de la votación en elecciones de diputados ${ }^{45}$.

Tabla 2. Resultados de las elecciones de diputados, 2017.

\begin{tabular}{|l|c|c|c|c|}
\hline & $\begin{array}{c}\text { Número de } \\
\text { votos }\end{array}$ & $\begin{array}{c}\text { Porcentaje } \\
\text { de votos }\end{array}$ & $\begin{array}{c}\text { Número de } \\
\text { escaños }\end{array}$ & $\begin{array}{c}\text { Porcentaje } \\
\text { de escaños }\end{array}$ \\
\hline Chile Vamos & 2.318 .719 & 38,7 & 72 & 46,5 \\
\hline La Fuerza de la Mayoría & 1.442 .196 & 24,1 & 43 & 27,7 \\
\hline Frente Amplio & 989.353 & 16,5 & 20 & 12,9 \\
\hline Convergencia Democrática & 640.612 & 10,6 & 14 & 9,0 \\
\hline Por Todo Chile & 235.576 & 3,9 & 1 & 0,6 \\
\hline Coalición Regionalista Verde & 115.323 & 1,9 & 4 & 2,6 \\
\hline Otros & 225.471 & 4,3 & 1 & 0,7 \\
\hline Total & 5.997 .250 & 100,0 & 155 & 100,0 \\
\hline
\end{tabular}

Fuente: Elaboración propia con datos del Servel.

44. TORO y VALENZUELA (2018) p. 225.

45. BUNKER (2018). 
En síntesis, las elecciones parlamentarias de 2017 muestran un salto significativo en la volatilidad electoral respecto a las tendencias que caracterizaron a la democracia chilena desde el retorno a la democracia ${ }^{46}$. Esto, según Cruz y Varetto podría deberse tanto a las modificaciones a las reglas electorales como a cambios en la identificación partidaria -especialmente en los más jóvenes-. En línea con lo indicado por Santana et $a l^{47}$, el aumento de la volatilidad electoral también supuso una acentuación de la fragmentación partidaria en el Congreso.

\section{Diseño de investigación y metodología}

El presente trabajo se propone explorar los efectos de distintos factores sobre el gasto electoral realizado por los candidatos en las elecciones para la Cámara de Diputados en Chile de 2017. Para esto se despliegan tres modelos de regresión lineal multivariable; esto permite captar el efecto de los factores independientes sobre una variable de salida continua, como es el gasto incurrido, los aportes de los partidos o el aporte propio. Los datos son los reportados públicamente por el Servicio Electoral, y que corresponden a 30.791 ingresos aportados a 939 candidaturas. La información permite desagregar por tipo de ingreso y su monto, permitiendo agregar los totales de cada ítem de ingreso por candidato. Los datos, una vez obtenidos, han sido procesado usando el software de análisis estadístico Stata.

Para el primer modelo, siguiendo a Navia y Acevedo ${ }^{48}$ se define como variable dependiente el gasto en función del límite máximo permitido. La legislación establece un límite de gasto electoral para cada distrito; la variable gasto entonces alude al porcentaje del gasto del candidato en cuestión, como parte del máximo permitido para su distrito. Tradicionalmente, se han usado el gasto por voto o el gasto total como referencia; sin embargo, el primero endogeniza la medición a la participación electoral, y el segundo lo endogeniza a lo que gastaron los otros candidatos en el distrito. Ya que el límite de gasto que la ley fija se define por la población del distrito, el gasto como proporción del máximo legalmente establecido permite obtener conclusiones relevantes a ese respecto.

Como variables explicativas se incluyen el aporte personal del candidato, el aporte del partido, la condición de incumbencia, densidad poblacional, participación electoral en el distrito para la elección de 2016, la coalición del candidato y el sexo.

Como se observa de la Tabla 1 las fuentes de financiamiento que para la elección de 2016 aumentaron su presencia respecto al gasto total son el aporte del partido y el

46. CRUZ Y VARETTO (2019).

47. SANTANA et al (2019).

48. NAVIA y ACEVEDO (2015). 
aporte propio del candidato. Respecto al primero, la legislación no establece límites a lo que el partido pueda aportar a la candidatura de su pacto, pudiendo llegar eventualmente al $100 \%$. En promedio, los candidatos recibieron aportes de sus partidos por el equivalente al $28,5 \%$ del gasto total permitido; hubo 20 candidaturas que se financiaron completamente con aportes del partido, y 136 que no recibieron ninguna donación por este concepto, lo que corresponde al 14,5\% del total. Esta variable se expresa como el porcentaje que representa el aporte del partido respecto a lo que efectivamente gastó cada candidatura.

En segundo lugar, la normativa dispone límites a lo que el candidato puede aportar a su propia campaña, que asciende al $25 \%$ del total de gasto permitido, con el objeto de reducir las brechas producto de asimetrías en las capacidades de gasto que los distintos candidatos puedan tener, evitando privilegios en la competencia para aquellos con más riqueza. Así, esta variable se traduce como el porcentaje que el aporte propio representó, sobre el total de aportes propios permitidos. Esta variable se constituye de forma distinta a la anterior, pues en aquella el aporte del partido se calcula como porcentaje respecto al total de gasto permitido. Por su parte, el aporte propio se considera como porción del máximo de aporte propio permitido por ley. A su vez el máximo de aporte propio permitido por la ley es del $25 \%$ del gasto total permitido. De esta forma, la variable "aporte propio" se calcula como porción de ese $25 \%$ del gasto, no del $100 \%$.

En promedio, los candidatos aportaron un 6,9\% del máximo permitido a sus propias campañas. Además, 276 candidatos de un total de 939 no realizaron aportes propios, lo que equivale al $29,4 \%$, y solo 27 candidatos superaron el $50 \%$ de lo permitido como aportes a su propia candidatura.

La condición de incumbencia se codifica como una variable dummy con valor o para los desafiantes y 1 para los 84 incumbentes. La densidad poblacional se expresa como la cantidad de habitantes por kilómetro cuadrado; para definir esta métrica, se recurrió a los datos del Sistema Nacional de Información Municipal (Sinim); ya que no existen datos sobre la densidad poblacional de cada distrito electoral, se agregaron los datos de las comunas que conforman cada distrito. Con ello se divide la sumatoria de la población en el distrito por los kilómetros cuadrados totales y se obtiene la métrica correspondiente al distrito. Lo propio se realiza con la participación electoral de 2016; ya que las elecciones fueron de carácter municipal, y los distritos son agregaciones de comunas, se agrupan los resultados por cada distrito y se obtiene su promedio. Sin embargo, es necesario matizar, puesto que existe importante variación en la asistencia a las urnas en las comunas dentro de un mismo distrito; pero para el candidato esto resulta indiferente puesto que no existe un patrón que concentre la votación a lo largo de los distritos, y esto lo fuerza a tener que hacer campaña en todas las comunas de su unidad electoral. 
Se incluye además la variable sexo, para controlar por potenciales diferencias en el esquema de financiamiento para hombres y mujeres.

Finalmente, se busca identificar diferencias en el gasto incurrido en función del pacto en el que compite el candidato. Para esto, la variable se codifica como categórica, teniendo como referencia al pacto Chile Vamos (valor $=0$ ), y se incluyen a Nueva Mayoría (=1), Convergencia Democrática $(=2)$, Frente Amplio $(=3)$ y Otros $(=4)$. Dada la gran cantidad de partidos que compiten conformando pactos electorales, resulta más funcional tomar la coalición más que el partido para identificar diferencias.

Adicionalmente, las variables aportes de partido (modelo 2) y aportes propios (modelo 3), que en el primero modelo se definieron como variables de entrada, son dispuestas como variables de salida, para determinar qué factores influyen en el financiamiento que candidatas y candidatos reciben de sus partidos o desde su propio "bolsillo".

En consecuencia, teniendo definidas las variables se proponen tres modelos lineales; el primero tiene al gasto como variable dependiente, el segundo al aporte del partido y el tercero a los aportes propios del candidato. Respecto al gasto, se espera que aquellas candidaturas que recibieron mayores aportes de sus partidos, y de los propios candidatos, desembolsen más en propaganda. Asimismo, parece plausible suponer que, en aquellos distritos más densamente poblados, y donde la participación en la elección pasada fue mayor, también se incurra en mayor gasto. Además, ya que los incumbentes tienen mayor acceso a recursos, eventualmente también desplegarían más propaganda.

En lo referente a los aportes de partido, se espera que los incumbentes reciban más donaciones desde el nivel central de sus estructuras partidarias, y que realicen más aportes de su propio bolsillo. Finalmente, se propone que los aportes propios estén positivamente relacionados con incumbencia. 


\section{Discusión de resultados}

Se despliegan tres modelos para evaluar la evolución de las variables:

Tabla 3. Modelos de regresión lineal para gasto, aportes de partido y aportes propios

\begin{tabular}{|l|c|c|c|}
\hline & $\begin{array}{c}\text { Modelo 1 } \\
\text { Gasto Total }\end{array}$ & $\begin{array}{c}\text { Modelo 2 } \\
\text { Aportes del Partido }\end{array}$ & $\begin{array}{c}\text { Modelo 3 } \\
\text { Aportes Propios }\end{array}$ \\
\hline APORTES DE PARTIDO & $-0,0520^{* * *}$ & & $-0,1290^{* * * *}$ \\
& $(0,0097)$ & & $(0,0162)$ \\
\hline APORTES PROPIOS & $0,4016^{* * * *}$ & $-0,4268^{* * * *}$ & \\
& $(0,0292)$ & $(0,0466)$ & \\
\hline INCUMBENTES & $9,0253^{* * * * *}$ & $-3,7962^{* *}$ & $9,7109^{* * *}$ \\
& $(1,8240)$ & $(1,8255)$ & $(2,7560)$ \\
\hline DENSIDAD & 0,0001 & $-0,0002$ & $-0,0000$ \\
& $(0,0001)$ & $(0,0001)$ & $(0,0001)$ \\
\hline PARTICIPACIÓN & 0,1125 & 0,1396 & $0,2165^{*}$ \\
& $(0,0624)$ & $(0,0981)$ & $(0,1014)$ \\
\hline COALICIÓN & & & $-1,6532$ \\
\hline NUEVA MAYORÍA & $-1,5377$ & $12,3136^{* * * *}$ & $(1,5615)$ \\
\hline CONVERGENCIA & $(1,041)$ & $(1,6972)$ & 1,9125 \\
DEMOCRÁTICA & $-7,2950^{* * * *}$ & 2,7041 & $(1,4197)$ \\
\hline FRENTE AMPLIO & $(1,0261)$ & $(2,1919)$ & $-8,5256^{* * * *}$ \\
& $-9,8095^{* * * *}$ & $-13,4857^{* * * *}$ & $(1,2316)$ \\
\hline OTROS & $(1,2438)$ & $(3,0299)$ & $-6,8025^{* * *}$ \\
& $-11,8430^{* * * *}$ & $-7,8803^{* * * *}$ & $(1,1915)$ \\
\hline SEXO & $(1,1876)$ & $(2,0299)$ & $-2,9901^{* * * *}$ \\
& $-1,7169^{* * * *}$ & $-0,8180$ & $(0,8214)$ \\
\hline & $(0,3472)$ & $(1,2595)$ & $\mathrm{N}=939$ \\
& $\mathrm{~N}=939$ & $\mathrm{~N}=939$ & $\mathrm{R} 2=0,2124$ \\
\hline & $\mathrm{R} 2=0,6249$ & $\mathrm{R} 2=0,1402$ & \\
\hline
\end{tabular}

Nota: *p $\leq 0.05,{ }^{* * *} \mathrm{p} \leq 0.01,{ }^{* * * *} \mathrm{p} \leq 0.001$. Errores estándar robustos por distrito entre paréntesis.

Fuente: Elaboración propia con datos del Servel y Sinim.

Todas las variables referentes a gasto y aportes son significativas entre sí, aunque sus coeficientes no son muy robustos (modelo 1). Sí parece haber más nitidez en la relación entre gasto e incumbencia, sexo, y coalición en los tres modelos. La variable participación está levemente correlacionada con los aportes propios (modelo 3). La variable de control densidad no tiene en absoluto incidencia en el gasto y los aportes.

De esta forma, los aportes del partido están negativamente correlacionados con el porcentaje del gasto máximo permitido y los aportes propios positivamente (mo- 
delo 1). Esto podría implicar que a medida que los candidatos más aportan a la campaña con sus propios fondos, menos dependientes se hacen del financiamiento que les provea su colectividad. Curiosamente, los aportes del partido, en tanto variable dependiente (modelo 2), están negativamente asociados a los aportes propios. Esto podría corroborar la noción de que los partidos aportan menos a quienes son más solventes para costear sus propias campañas. O, que los candidato/as contribuyen más personalmente cuando los partidos parecen no tener mucho interés en hacerlo. Finalmente, los aportes propios van en la misma dirección, puesto que correlacionan negativamente con los aportes de partido. Es necesario insistir en que la magnitud de los coeficientes es más bien débil, por lo que estos resultados deben tomarse con cautela.

Sí parece haber más claridad respecto a la relación entre incumbencia, gasto y los aportes. En efecto, los incumbentes gastaron en promedio nueve puntos porcentuales más que los desafiantes, controlando por las demás variables (modelo 1). Asimismo, los incumbentes también aportaron en promedio nueve puntos porcentuales más a sus propias campañas, lo que podría explicarse por los sustantivos ingresos que les significa la dieta parlamentaria (modelo 3). Sorpresivamente, el factor incumbencia está asociado negativamente con los aportes de los partidos (modelo 2); es decir, los partidos aportan menos a los incumbentes que a los desafiantes. Esto va en la línea de lo mencionado respecto a la relación entre aportes de partidos y aportes propios: ya que los incumbentes realizarían más aportes a sus propias campañas (ver figura 1), los partidos parecen distribuirles menos recursos, asumiendo que los incumbentes tienen más solvencia. La Figura 1 muestra el promedio de gasto para incumbentes y desafiantes, evidenciando que el patrón de mayor gasto promedio en incumbentes se repite para todos los pactos:

Figura 1. Diferencia en el gasto entre desafiantes e incumbentes, por pacto.

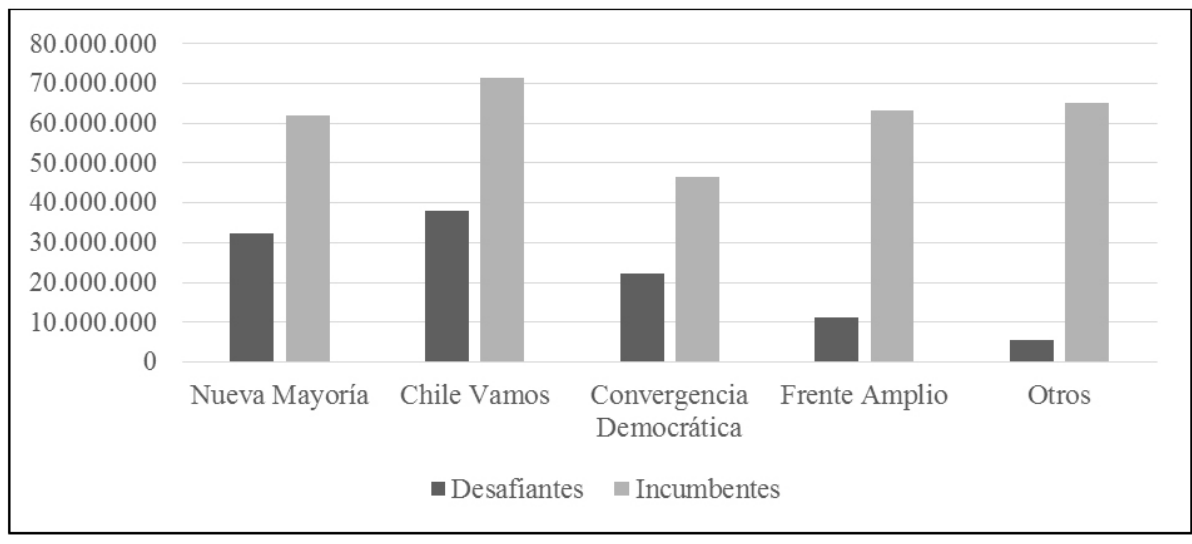

Fuente: Elaboración propia. 
Llama la atención la marcada diferencia entre los incumbentes del Frente Amplio y "otros", respecto a los desafiantes de sus mismos pactos (figura 1). En total, son cinco los incumbentes que se inscriben en esa categoría; tres para el Frente Amplio y dos independientes. Esto no implica per se que las candidaturas del Frente Amplio gasten más -si no, así lo mostraría el análisis de regresión-, sino que el hecho de que el número de observaciones para aquellos casos sea tan menor respecto al resto de coaliciones, implica que el promedio no es "arrastrado" hacia abajo por la dispersión de los datos, lo que sí ocurre con las coaliciones grandes en las que hay más observaciones, y por tanto más varianza.

Muchos de los argumentos en contra de la reforma apuntaban a que limitar el gasto permitido favorecería a los incumbentes; al parecer, efectivamente el nuevo esquema propició un mayor gasto de los incumbentes, pero eso no necesariamente se tradujo en un mayor éxito electoral para los mismos: "aquello sucedió en la elección municipal, donde se incrementó la reelección de los alcaldes que volvieron a competir, pero no se replicó esta ventaja de incumbentes en las elecciones de congresistas de $2017^{\prime 49}$. El análisis estadístico sí nos muestra que los incumbentes gastan más que los desafiantes (modelo 1), reciben menos aportes desde sus partidos (modelo 2), y tienden a contribuir más de sus propios bolsillos (modelo 3).

La variable sexo arroja significancia estadística en los modelos 1 y 3: las mujeres, en promedio, gastaron un poco menos que los hombres, pero esto no se replica respecto a los aportes de los partidos, donde no hay diferencia. Sí se vislumbra una diferencia más significativa respecto a los aportes propios, pues las mujeres parecen incurrir menormente en gastos a su propio cargo que los hombres. Este hallazgo es interesante puesto que, si las mujeres incurren en menos gasto propio, y como consecuencia incurren en menos gasto en propaganda, pero los aportes de los partidos no diferencian el sexo del candidato, entonces los partidos no están actuando como promotores de campañas más intensivas para las mujeres. La ley de financiamiento electoral propone un incentivo a los partidos, puesto que les entrega un aporte extraordinario como "premio" por cada candidata electa, pero no parece incidir en las decisiones de gasto de las colectividades.

Sí hay asociación estadística más sustantiva cuando se trata de examinar el efecto de las coaliciones. En lo referente al gasto, ya que la categoría de referencia es la coalición Chile Vamos, y la asociación con la categoría Nueva Mayoría es nula, se infiere que en promedio las candidaturas de Chile Vamos no se diferencian de las de la Nueva Mayoría en la magnitud del gasto por candidato (modelo 1). Sin embargo, es en lo relativo a Convergencia Democrática, Frente Amplio y los demás pactos menores donde la asociación es sustantivamente más significativa, en orden negativo, ya que gastaron aproximadamente entre 7 y 11 puntos porcentuales menos que Chile Vamos (modelo 1).

49. FUENTES (2018) p. 133. 
Llama la atención la robustez de los resultados respecto a los aportes desde los partidos (modelo 2). Todas las coaliciones, a excepción de Convergencia Democrática, registraron un flujo distinto de aportes desde los partidos hacia las candidaturas en relación con Chile Vamos. Se observa que los partidos de la Nueva Mayoría son más activos en distribuir aportes hacia sus candidatos, y que las campañas del Frente Amplio y las demás coaliciones recibieron a su vez aportes menores que Chile Vamos, y sobre todo menores que los candidatos de la Nueva Mayoría. La Figura 2 muestra la distribución de las candidaturas en relación con la cuantía del aporte recibido desde sus partidos, respecto a lo efectivamente gastado. Una hipotética relación lineal mostraría que el gasto incurrido aumenta proporcionalmente respecto a lo recibido desde el partido; este no parece ser el caso. Mas bien, en la mayor parte de los datos el aporte de los partidos representa menos del $20 \%$ del gasto permitido, al tiempo que el gasto efectivo usualmente llegó hasta no más del 50\%. Esto implica que las candidaturas se valieron de otras fuentes de ingresos, y que el aporte de los partidos no necesariamente es el componente más sustantivo de sus ingresos.

Figura 2. Aporte del partido como componente del gasto, desafiantes e incumbentes.

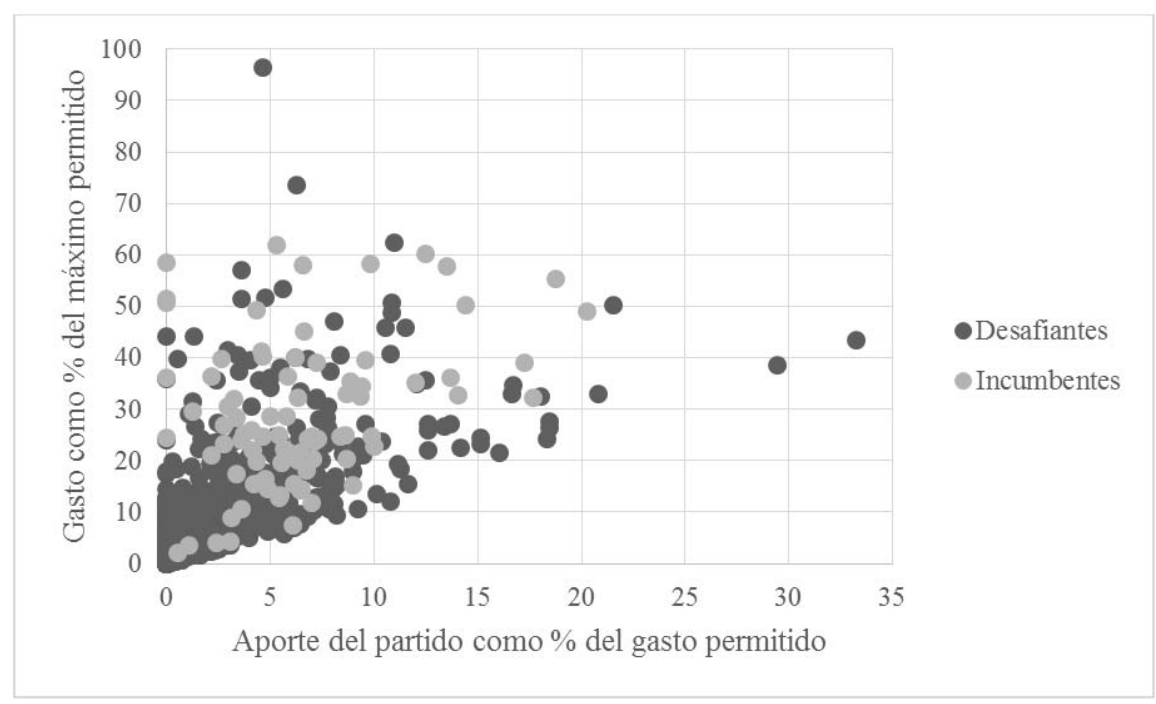

Fuente: Elaboración propia con datos del Servel.

Otro dato interesante es que la coalición también hace una diferencia respecto a la magnitud del aporte propio. No se vislumbran distinciones entre Chile Vamos y la Nueva Mayoría, pero los candidatos del Frente Amplio y los pactos menores desembolsan menos recursos propios para sus campañas, puesto que también gastan menos. 
La estructura de financiamiento es relativamente similar a lo acaecido para las elecciones anteriores (ver Tabla 1); llama la atención que para la elección de diputados de 2017 la agregación del aporte personal de los candidatos sea menor que para las municipales de 2016. De hecho, este último está más lejos del máximo legal de aportes propios (25\%), lo que puede explicar la mayor presencia de los aportes de personas en el esquema de financiamiento de la elección de diputados. Además, los partidos por sí mismos también pueden hacer campaña.

Tabla 4. Financiamiento de campañas de diputados, 2017.

\begin{tabular}{|l|l|}
\hline Aportes de los partidos & 26,7 \\
\hline Aportes de personas & 27,2 \\
\hline Aporte propio & 18,4 \\
\hline Aporte fiscal & 27,6 \\
\hline
\end{tabular}

Fuente: Elaboración propia con datos del Servel.

En lo referente a población (densidad), los datos no muestran ninguna relevancia estadística a este efecto. Se rechaza por tanto la presunción de que las candidaturas gastan más en los lugares densamente poblados. Una explicación plausible a este respecto la entregan Toro y Valenzuela, quienes sugieren que "para enfrentar distritos más grandes se utilizó la segmentación territorial dentro de los pactos, a través de la división de sectores entre compañeros de lista (...) las votaciones de los candidatos en cada comuna no fueron homogéneas y dependieron mucho de la capacidad territorial de cada uno de los candidatos"50. En cambio, la participación en la elección de 2016 sí se halla positivamente asociada, aunque muy levemente, con los aportes propios. Esto podría llevar a suponer que los candidatos, conscientes de la participación en su distrito, adicionan más recursos para desplegar propaganda; sin embargo, como el gasto no está asociado con la participación no está clara la relación entre ambas variables. De todas maneras, el coeficiente no arroja mayor consistencia y la significancia estadística no sugiere una asociación muy marcada ${ }^{51}$.

50. TORO y VALENZUELA (2018) p. 229.

51. Un análisis de robustez presentado en el anexo, para el cual se utilizanregresiones de conteonegativo binomial-para controlar por el alto número de ceros, nos muestra que los resultados no cambian dramáticamente. En ambos tipos de análisis estadístico, la variable dependiente es continua. Y, aunque la regresión binominal negativa asume variables discretas, en este último las variables dependientes asumen solo valores no negativos y su distribución se asimila a la distribución de conteo de tipo negativa negativa binomial. 


\section{Conclusiones}

La elección tuvo importantes consecuencias para el funcionamiento del sistema político. Los partidos de la Coalición Chile Vamos aumentaron su presencia parlamentaria, entraron nuevas fuerzas políticas al Congreso que ante la fórmula binominal estaban impedidas de tener mayor representación parlamentaria, y disminuyó la propaganda electoral.

Se presentaron tres modelos de regresión lineal para estimar el efecto de distintas variables explicativas en el nivel de gasto de las candidaturas. Para esto, a diferencia de trabajos anteriores, se tomó el porcentaje de gasto incurrido en función del máximo permitido, como variable dependiente (modelo 1). Adicionalmente, se desplegaron modelos con el aporte de los partidos (modelo 2) y el aporte propio (modelo 3), como variables dependientes. Respecto al gasto (modelo 1), se obtiene que los incumbentes fueron más proclives al gasto electoral que los desafiantes; esto no necesariamente implica que en términos brutos hayan gastado más - puesto que son menos numerosos-, pero sí que cuando despliegan sus candidaturas suelen acercarse más al máximo de gasto permitido que les impone la ley. Asimismo, en línea con lo observado en trabajos anteriores ${ }^{52}$, las candidata/os de la coalición de derecha Chile Vamos, en general, realizaron un gasto mayor de acuerdo con el máximo permitido (modelo 1), tendieron a recibir más aportes de sus partidos (modelo 2), y desembolsaron más recursos personales que aquellas del Frente Amlio y otros pactos (modelo 3).

Respecto a los aportes de partidos, destaca el hecho que los candidatos de la Nueva Mayoría financiaron sus campañas en buena medida con donaciones de sus partidos, o al menos en mayor medida que el resto de las coaliciones. El Frente Amplio y otras coaliciones menores recurrieron mucho menos al financiamiento desde los partidos para cubrir los gastos de propaganda. Sorpresivamente, los partidos no asignaron recursos estratégicamente a sus candidatos en función de la participación en la elección anterior o densidad poblacional del distrito. Inicialmente, se esperaba que los incumbentes recibieran más aportes de partido, y además contribuyeran más desde sus propios fondos. Pero los resultados solo respaldan esta hipótesis en cuanto al gasto propio (modelo 3), y nos muestran lo opuesto respecto de los aportes de los partidos (modelo 2).

La magnitud del financiamiento propio está asociada negativamente con los aportes de partido por lo que quienes recurren más a sus propios fondos, son menos dependientes del financiamiento de los partidos. Sin embargo, los coeficientes que dan cuenta de dicha asociación no son tan robustos. Lo que sí es más nítido es que los incumbentes incurrieron en mayores aportes propios, y que los candidatos del Frente

52. JOHNSON (2011); GONZÁLEZ et al (2015). 
Amplio y las demás coaliciones menores a su vez realizaron aportes sustantivamente menores a sus propias candidaturas, en relación con Chile Vamos.

La noción de que los candidatos incumbentes gastan más que los desafiantes (modelo 1) es coincidente con otros trabajos en la materia ya señalados anteriormente ${ }^{53}$. Además, el mayor gasto de la coalición de derecha también es convergente con algunos de los hallazgos anteriores ${ }^{54}$.

Finalmente, respecto al género de las candidaturas, se identifican algunos hallazgos interesantes. En promedio las candidaturas de mujeres incurren en menos gasto que la de los hombres (modelo 1), y realizan algo menos de aportes propios (modelo 3). Sin embargo, no hay distinción respecto a los aportes de partido (modelo 2). Esto implicaría que los partidos no estarían desplegando acciones de promoción de las candidaturas de mujeres, o al menos no en lo referente al financiamiento de las campañas.

\section{Referencias bibliográficas}

ABBE, Owen J. y HERNSONN, Paul S. (2003): “Campaign Professionalism in State Legislative Elections", State Politics and Policy Quarterly, Vol. 3, $\mathrm{N}^{\circ}$, septiembre de 2003, pp. 223-245.

ABRAMOWITZ, Alan I. (1988): "Explaining Senate Election Outcomes”, The American Political Science Review, Vol. 82, N² 2, junio de 1988, pp. 385-403.

ABRAMOWITZ, Alan I. (1991): "Incumbency, Campaign Spending, and the Decline of Competition in U.S. House Elections", The Journal of Politics, Vol. 53, $\mathrm{N}^{\circ} 1$, febrero de 1991, pp. 34-56.

AGOSTINI, Claudio (2012): "Financiamiento de la política en Chile. Campañas electorales de 2009-2010". En DÍAZ, Francisco y SIERRA, Lucas . Democracia con Partidos. Informe para la reforma de los partidos políticos en Chile (Santiago, CEP/ CIEPLAN), pp. 269-319.

ALTMAN, David (2016): "Informe Especial: La caida del Binominal". En LUNA, Juan Pablo; TORO, Sergio; JARAMILLO-BRUN, Nathalie y ACEVEDO, Carolina. En Cultura política de la democracia en Chile y en las Américas, 2014: ¿Tiempo de reformas? (Nashville, Vanderbilt University, PUC), p. 127.

53. DÍAZ RIOSECO et al (2006) AGOSTINI (2012); MORALES Y PIÑEIRO (2010); NAVIA E ITURRA (2016).

54. DÍAZ RIOSECO et al (2006); AGOSTINI (2012). 
ANDERSON, Malcom y THAM, Joo-Cheong (2014): "Dynamics of Electoral Expenditure and the 'arms Race' Thesis: The Case of New South Wales", Australian Journal of Political Science, Vol. 49, $\mathrm{N}^{\circ}$ 1, febrero de 2014, pp. 84-101.

ABRAMS, Burton A. y SETTLE, Russell F. (2004): "Campaign-Finance Reform: A Public Choice Perspective”, Public Choice, Vol. 120, $\mathrm{N}^{\circ}$, septiembre de 2004, pp. 379-400.

BENOIT, Kenneth y MARSH, Michael (2008): “The Campaign Value of Incumbency: A New Solution to the Puzzle of Less Effective Incumbent Spending", American Journal of Political Science, Vol. 52, N 4, octubre de 2008, pp. 874-89o.

BEONIT, Kenneth Y MARSH, Michael (2010): "Incumbent and Challenger Campaign Spending Effects in Proportional Electoral Systems: The Irish Elections of 2002", Political Research Quarterly, Vol. 63, N 1, marzo de 2008, pp. 159-173.

BEN-BASSAT, Avi, DAHAN, Momi y KLOR, Esteban F. (2015): "Does Campaign Spending Affect Electoral Outcomes?", Electoral Studies, Vol. 40, diciembre de 2015, pp. 102-14.

BUNKER, K. (2018): "La elección de 2017 y el fraccionamiento del sistema de partidos en Chile", Revista Chilena de Derecho y Ciencia Política, Vol. 9, $\mathrm{N}^{\circ}$ 2, diciembre 2018, pp. 204-229.

BUTCHER, Jordan y MILYO, Jeffrey (2020): “Do Campaign Finance Reforms Insulate Incumbents from Competition? New Evidence from State Legislative Elections", PS: Political Science E Politics, Vol. 53, $\mathrm{N}^{\circ}$ 3, septiembre de 2020, pp. 460-464.

CASTAÑEDA, Néstor (2018): "Electoral Volatility and Political Finance Regulation in Colombia”, Colombia Internacional, Vol. 95, julio-septiembre 2018, pp. 3-24.

CRUZ, Facundo y VARETTO, Carlos (2019): "Crónica de un cambio anunciado. Las elecciones de 2017 en Chile frente al cambio de sistema electoral", Estudios Políticos, Vol. 54, enero de 2019, pp. 233-258.

DÍAZ RIOSECO, Diego; GIANNINI, Pilar; LUNA, Juan Pablo, y NÚÑEZ, Rodrigo (2006): "El secreto de mi éxito. seis caminos para llegar y permanecer en Valparaíso", Revista de Ciencia Política, Vol. 26, pp. 169-19o.

ERIKSON, Robert S. y PALFREY, Thomas R. (1998): "Campaign spending and incumbency: An alternative simultaneous equations approach", Journal of Politics, Vol. $60, \mathrm{~N}^{\circ} 2$, mayo de 1988, pp. 355-373.

EVANS, Thomas A. (2007): "An Empirical Test of Why Incumbents Adopt Campaign Spending Limits", Public Choice, Vol. 132, $\mathrm{N}^{\circ}$ 3, mayo de 2007, pp. 437-56. 
FIGUEROA-HUENCHO, Verónica; OLAVARRÍA, Mauricio y NAVARRETE, Bernardo (2011): "Política de Modernización de la Gestión Pública en Chile 19902006: evidencias a partir de un modelo de análisis", Convergencia, $\mathrm{N}^{\circ} 57$, septiembre-diciembre de 2011, pp. 61-99.

FINK, Alexander (2012): "The Effects of Party Campaign Spending under Proportional Representation: Evidence from Germany", European Journal of Political Economy, Vol. 28, $\mathrm{N}^{\circ}$ 4, diciembre de 2012, pp. 574-92.

FUENTES, C. (2018): "Cortándose las alas. Factores que explican la reforma al financiamiento electoral en Chile", Colombia Internacional, Vol. 95, julio-septiembre de 2008, pp. 109-136.

GAMBOA, Ricarado y MORALES, Mauricio (2016): "Chile's 2015 Electoral Reform: Changing the Rules of the Game", Latin American Politics and Society, Vol. 58, octubre de 2016, pp. 126-144.

GAMBOA, Ricardo y SEGOVIA, Carolina (2016): "Chile 2015: Falla política, desconfianza y reforma", Revista de Ciencia Política, Vol. 36, № 1, pp. 123-144.

GERBER, Alan (1998): "Estimating the Effect of Campaign Spending on Senate Election Outcomes Using Instrumental Variables", The American Political Science Review, Vol. 92, $\mathrm{N}^{\circ}$ 2, junio de 1988, pp. 401-411.

GONZÁLEZ, Ricardo; SIERRA, Lucas y SZEDERKENYI, Francisco (2015): "La práctica del financiamiento privado en las elecciones parlamentarias", Puntos de Referencia (Centro de Estudios Públicos), N 394, marzo de 2015.

GREEN, Donald P. y KRASNO, Jonathan S. (1988): "Salvation for the Spenthrift: reestimating the effects of campaign spending in house elections", American Journal of Political Science, Vol. 32, $\mathrm{N}^{\circ}$ 4, noviembre de 1988, pp. 884-907.

HALL, Melinda Gall y BONNEAU, Chris W. (2008): "Mobilizing Interest: The Effects of Money on Citizen Participation in State Supreme Court Elections", American Journal of Political Science, Vol. 52, $\mathrm{N}^{\circ}$ 3, julio de 2008, pp. 457-470.

HOGAN, Robert E. (200o): "The Costs of Representation in State Legislatures: Explaining Variations in Campaign Spending", Social Science Quarterly, Vol. 81, $\mathrm{N}^{\circ}$ 4, diciembre 2000, pp. 941-956.

HOGAN, Robert E. (2007): “The Effects of Candidate Gender on Campaign Spending in State Legislative Elections", Social Science Quarterly, Vol. 88, ${ }^{\circ}{ }_{5}$, diciembre de 2007, pp. 1092-105.

JACOBSON, Gary C (1978): “The effects of campaign spending in congressional elections", American Political Science Review, Vol. 72, N 2, junio de 1978, pp. 469-91. 
JACOBSON, Gary C. (1980): Money in congressional elections (New Haven: CT, Yale University Press).

JACOBSON, Gary C. (1984): “Money in the 1980 and 1982 congressional elections”. En MALBIN, Michael J. . Money and politics in the United States: Financing elections in the 1980s (Chatham: NJ, Chatham House).

JACOBSON, Gary C. (1985): "Money and Votes Reconsidered: Congressional Elections, 1972-1982", Public Choice, Vol. 47, N 1, enero de 1985, pp. 7-62.

JACOBSON, Gary C. (1990): “The Effects of Campaign Spending in House Elections: New Evidence for Old Arguments", American Journal of Political Science, Vol. 34, $\mathrm{N}^{\circ}$ 2, mayo de 1990, pp. 334-362.

JOHNSON, Joel W. (2011): "Incumbents without a Campaign Finance Advantage: Competition and Money in Chile's Congressional Elections", Journal of Politics in Latin America, Vol. 3, $\mathrm{N}^{\circ}$ 3, pp. 3-33.

JOHNSON, Joel W. (2012): "Campaign Spending in Proportional Electoral Systems: Incumbents Versus Challengers Revisited”, Comparative Political Studies, Vol. 46, $\mathrm{N}^{\circ}$ 8, octubre de 2012, pp. 968-93.

MADDENS, Bart. y PUT, Gert-Jan (2013): "Office Effects and Campaign Spending in a Semi-open List PR System: The Belgian/Flemish Federal and Regional Elections 1999-2010", Electoral Studies, Vol. 32, N 4, diciembre de 2013, pp. 852-63.

MORALES, Mauricio y PIÑEIRO, Rafael (2010): "Gasto en campaña y éxito electoral de los candidatos a diputados en Chile 2005", Revista de Ciencia Política, Vol. 30, $\mathrm{N}^{\circ} 3$, pp. $645-667$.

MOON, Woojin (2006): “The Paradox of Less Effective Incumbent Spending: Theory and Tests". British Journal of Political Science", Vol. 36, $\mathrm{N}^{\circ}$ 4, octubre de 2006, pp. 705-721.

NAVIA, Patricio y ACEVEDO, Sergio (2015): "Un método no endógeno para medir el gasto electoral en Chile 2005-2009”. Revista Mexicana de Ciencias Políticas y Sociales, Vol. 60, N²25, septiembre-diciembre de 2015, pp. 111-138.

NAVIA, Patricio e ITURRA, Pamela (2016): "Los determinantes de los componentes del gasto electoral en elecciones municipales en Chile, 2008-2012", Revista Chilena de Derecho y Ciencia Política, Vol. $7, \mathrm{~N}^{\circ}$ 3, septiembre-diciembre de 2016, pp. 57-90.

OLAVARRÍA-GAMBI, Mauricio; NAVARRETE, Bernardo y FIGUEROA-HUENCHO, V. (2011): “¿Cómo se formulan las políticas públicas en Chile?”, Política y Gobierno, Vol. 18, $1^{\circ}$ semestre de 2011, pp. 109-154. 
OLAVARRÍA-GAMBI, Mauricio (ed.) (2012): ¿Cómo se formulan las políticas públicas en Chile?. Tomo 1, La modernización de la gestión pública (Santiago de Chile, Editorial Universitaria).

RAMOS, Conrado y SCROLLINI, Fabrizio (2013): "Los nuevos acuerdos entre políticos y servidores públicos en la alta dirección pública en Chile y Uruguay", Revista Uruguaya de Ciencia Política, Vol. 22, $\mathrm{N}^{\circ}$ 1, diciembre de 2013, pp. 11-36.

SANTANA, Andrés; MONTERO, José Ramón y RAMA, José (2019): "Las elecciones parlamentarias chilenas de 2017: características de los votantes y factores de voto", América Latina Hoy, Vol. 81, marzo de 2019, pp. 147-171.

SCARROW, Susan (2004): "Explaining Political Finance Reforms: Competition and Context”, Party Politics, Vol. 10, N6, noviembre de 2004, pp. 653-75.

SHIN, Myungsoon; JIN, Youngjae; GROSS, Donald A. y EOM, Kihong (2005): "Money Matters in Party-centered Politics: Campaign Spending in Korean Congressional Elections", Electoral Studies, Vol. 24, № 1, marzo de 2005, pp. 85-101.

SPARKS, Steven (2018): "Campaign spending and the top-two primary: How challengers earn more votes per dollar in one-party contests", Electoral Studies, Vol. 54, agosto 2018, pp. 56-65.

SPRICK, Steven (2020): "Does Campaign Spending Affect Election Outcomes? New Evidence from Transaction-Level Disbursement Data", The Journal of Politics, Vol. $82, \mathrm{~N}^{\circ} 4$, octubre de 2020, pp. 1502-1515.

TELLO, Felipe (2011): “La política de reforma y modernización de la gestión pública en Chile. Actores y procesos", Universum, Vol. 2, $\mathrm{N}^{\circ}$ 26, pp. 245-265.

TORO, Sergio y VALENZUELA, Macarena (2018): "Chile 2017: ambiciones, estrategias y expectativas en el estreno de las nuevas reglas electorales", Revista Ciencia Política, Vol. 38, $\mathrm{N}^{\circ}$ 2, pp. 207-232.

WEINSCHENK, Aaron C. y HOLBROOK, Thomas M. (2014): “The Determinants of Campaign Spending in Mayoral Elections", State and Local Government Review, Vol. 46, $\mathrm{N}^{\circ}$ 1, marzo de 2014, pp. 13-27. 
Anexo 1. Modelos de regresión binominal negativa para aportes del partido y aportes propios

\begin{tabular}{|c|c|c|}
\hline & APORTES DEL PARTIDO & APORTES PROPIOS \\
\hline APORTES DE PARTIDO & & $\begin{array}{c}-0,0406^{* * *} \\
(0,0044)\end{array}$ \\
\hline APORTES PROPIOS & $\begin{array}{l}-0,163^{* * *} \\
(0,0022)\end{array}$ & \\
\hline INCUMBENTES & $\begin{array}{l}-0,1132 \\
(0,0716)\end{array}$ & $\begin{array}{c}0,6486 \% * \% \\
(0,1524)\end{array}$ \\
\hline DENSIDAD & $\begin{array}{l}-0,0000 \\
(0,0001)\end{array}$ & $\begin{array}{l}-0,0000 \\
(0,0001)\end{array}$ \\
\hline PARTICIPACIÓN & $\begin{array}{c}0,0064 \\
(0,0046)\end{array}$ & $\begin{array}{l}0,0401 * \% \\
(0,0168)\end{array}$ \\
\hline NUEVA MAYORÍA & $\begin{array}{c}0,394 * * * \% \\
(0,047)\end{array}$ & $\begin{array}{l}0,3501^{*} \\
(0,1534)\end{array}$ \\
\hline $\begin{array}{l}\text { CONVERGENCIA } \\
\text { DEMOCRÁTICA }\end{array}$ & $\begin{array}{c}0,083 \\
(0,0691)\end{array}$ & $\begin{array}{c}0,0240 \\
(0,1137)\end{array}$ \\
\hline FRENTE AMPLIO & $\begin{array}{c}-0,5181^{* * * *} \\
(0,1369)\end{array}$ & $\begin{array}{c}-1,8875^{* * *} \\
(0,2532)\end{array}$ \\
\hline OTROS & $\begin{array}{c}-0,2728^{* * * *} \\
(0,079)\end{array}$ & $\begin{array}{c}-1,7599 * * * \\
(, 2059)\end{array}$ \\
\hline \multirow[t]{2}{*}{ SEXO } & $\begin{array}{l}-0,0294 \\
(0,541)\end{array}$ & $\begin{array}{c}-0,4922^{* * * *} \\
(0,1166)\end{array}$ \\
\hline & $\begin{array}{c}\mathrm{N}=939 \\
\mathrm{R} 2=0,1603\end{array}$ & $\begin{array}{c}\mathrm{N}=939 \\
\mathrm{R} 2=0,4498\end{array}$ \\
\hline
\end{tabular}

Nota: ${ }^{*} \mathrm{p} \leq 0.05,{ }^{* * *} \mathrm{p} \leq 0.01,{ }^{* * * *} \mathrm{p} \leq 0.001$. Errores estándar robustos por distrito entre paréntesis 